ISSN = 1980-993X - doi:10.4136/1980-993X
www.ambi-agua.net
E-mail: ambi-agua@agro.unitau.br
Tel.: (12) 3625-4212

\title{
Avaliação da integridade ecológica de rios em áreas do zoneamento ecológico econômico do complexo hidrográfico Guapiaçu-Macacu, RJ, Brasil
}

\author{
(http://dx.doi.org/10.4136/ambi-agua.762) \\ Priscilla da Silva Pereira ${ }^{1}$; Lia Amorim Chaves Fernandes ${ }^{1}$; Jaime Lopes da Mota \\ Oliveira $^{2}$; Darcilio Fernandes Baptista ${ }^{1}$ e-mail: pris.pereira@ioc.fiocruz.br; lia_acf@ioc.fiocruz.br; e-mail: darcilio@ioc.fiocruz.br;
${ }^{2}$ Departamento de Saneamento e Saúde Ambiental/ENSP/FIOCRUZ e-mail: jaimel@ensp.fiocruz.br. \\ ${ }^{1}$ Laboratório de Avaliação e Promoção da Saúde Ambiental/IOC/FIOCRUZ
}

\section{RESUMO}

Os ecossistemas aquáticos têm sido frequentemente alterados de maneira significativa por múltiplos impactos. O complexo hidrográfico Guapiaçu-Macacu é uma importante bacia do Rio de Janeiro com zonas ecológicas distintas propostas no Zoneamento Ecológico Econômico. Esse estudo avaliou a integridade ecológica em trechos de rios deste Complexo localizados na Zona de Conservação da Vida Silvestre (ZCVS) e Zona de Uso Agropecuário (ZUAP) por meio do Protocolo de Avaliação Visual (PAV) e das variáveis físicas, químicas e microbiológicas. A integração dos resultados mostrou uma diferença significativa entre os pontos de menor grau de contaminação na ZCVS e os trechos com maior grau de impacto na ZUAP. O PAV foi mais sensível que os parâmetros convencionais entre os trechos ecologicamente impactados e os intermediários localizados na ZUAP. Esse tipo de avaliação mostrou ser eficiente no monitoramento ambiental de bacias hidrográficas que tenham um plano de Zoneamento Ecológico Econômico. Portanto, a utilização de métodos físicos, químicos e microbiológicos pode ser complementada com o PAV.

Palavras-chave: Avaliação da qualidade da água; protocolo de avaliação visual; zoneamento ecológico-econômico.

\section{Assessment of water quality in areas of ecological economic zoning of the Guapiaçu-Macacu basin, RJ, Brazil}

\begin{abstract}
Aquatic ecosystems have often been significantly altered by multiple impacts. The Guapiaçu-Macacu Hydrographic Complex is an important basin in Rio de Janeiro characterized by distinct ecological zones that make up an Ecological Economic Zoning. This research evaluated ecological upright in segments of this Complex located in Wildlife Conservation Zone (WCZ) and the Agricultural Use Zone (AUZ) using the Protocol Visual Assessment (PVA) and physical, chemicals and microbiology methods. The results showed a significant difference between the points of lowest contamination degree in WCZ and stretches with a greater impact degree in AUZ. The PVA was more sensible than the conventional parameters in the resolution between segments impacted environmentally and
\end{abstract}


PEREIRA, P. S.; FERNANDES, L. A. C.; OLIVEIRA, J. L. M.; BAPTISTA, D. F. Avaliação da integridade ecológica de rios em áreas do zoneamento ecológico econômico do complexo hidrográfico Guapiaçu-Macacu, RJ, Brasil. Ambi-Agua, Taubaté, v. 7, n. 1, p. 157-168, 2012. (http://dx.doi.org/10.4136/ambi-agua.762)

impacted middle located in AUZ. This type of evaluation proved to be more effective in environmental monitoring the water quality for watersheds that have their Ecological Economic Zoning Plan. Therefore, the use of physical, chemical and microbiological methods must be complemented by the PVA.

Keywords: Assessment of water quality; protocol visual assessment; ecological economic zoning.

\section{INTRODUÇÃO}

Os ecossistemas aquáticos têm sido frequentemente alterados de maneira significativa pelo uso inadequado do solo, podendo provocar a redução na qualidade da água para o consumo humano e integridade biológica (Machado e Klein, 2003; Tundisi, 2003; Pedreira et al., 2009). Uma das principais intervenções ocorre devido à retirada das matas ciliares, aumentando a velocidade da correnteza, o que contribui para o arrasto de sedimentos, acelerando o processo de assoreamento e erosão (Tucci, 2002; Santos e Cardoso, 2007; Sarcinelli et al., 2008, Carvalho et al., 2000). Em geral, a qualidade da água de um rio é determinada pela medição de parâmetros físicos, químicos e microbiológicos (Coradi et al., 2009, Campanha et al., 2010). No entanto, Rodrigues et al. $(2008,2010)$ sugerem a avaliação da paisagem do rio e de seu entorno como ferramenta complementar para um diagnóstico da qualidade do ecossistema aquático por meio da aplicação do Protocolo de Avaliação Visual (PAV).

A ocupação e o manejo do solo e a exploração dos recursos naturais devem adotar como base a biodiversidade, a qualidade e a manutenção dos ecossistemas locais. Para nortear esse processo, normalmente o ambiente é dividido em áreas que compõe um Zoneamento Ecológico Econômico (ZEE). Um ZEE consiste na elaboração do diagnóstico das potencialidades do patrimônio ambiental e sociocultural de um determinado espaço geográfico (Ab'Saber, 1989; Acselrad, 2000). A proposta de ZEE para a bacia GuapiaçuMacacu é composta por sete zonas distintas definidas por um conjunto de regras e orientações para os diferentes usos da terra, produzindo uma sequência de mosaico que fragmentam a paisagem como mostra a Figura 1 (Cabral e Fiszon, 2004). A Zona de Preservação da Vida Silvestre (ZPVS) e a Zona de Conservação da Vida Silvestre (ZCVS) são as áreas de uso mais restritivo da terra por apresentarem unidades de conservação como parques e reservas. As áreas com menor ou nenhuma restrição quanto ao uso da terra são: Zona de Ocupação Controlada (ZOC) onde existem cidades e/ou vilas; Área de Uso Conflitante (AUC) com condições ambientais que favorecem as atividades de desenvolvimento; Zona de Uso Agropecuário (ZUAP) que possui atividades agrícolas e pecuárias; Zona de Uso Especial (ZUE) onde será instalado o Complexo Petroquímico da Petrobras (COMPERJ); e Área Crítica que está composta por uma planície inundável com pequenas atividades agropecuárias.

Desse modo, o monitoramento é fundamental para garantir a integridade ecológica, a manutenção da vida selvagem e a saúde da população usuária dessas águas. Esse estudo teve como objetivo avaliar a integridade ecológica em trechos de rios localizados em duas ZEEs (ZCVS e ZUAP) na sub-bacia do rio Guapiaçu usando as variáveis físicas, químicas, microbiológicas e do Protocolo de Avaliação Visual (PAV). Além disso, foi verificada a importância do emprego do PAV como ferramenta em programas de monitoramento de águas superficiais. 


\section{MATERIAIS E MÉTODOS}

\subsection{Descrição da área de estudo}

O Complexo Hidrográfico Guapiaçu-Macacu possui uma área de drenagem de cerca de $1.640 \mathrm{Km}^{2}$, com uma população do entorno acima de 100 mil habitantes. Suas águas atendem cerca de 2,5 milhões habitantes com irrigação, piscicultura, agropecuária e abastecimento público dos municípios de Cachoeira de Macacu, Guapimirim, Itaboraí, São Gonçalo e Niterói (JICA, 1994; Helder, 1999). Em função da importância deste sistema hidrográfico para o estado do Rio de Janeiro, foi criada a Área de Proteção Ambiental (APA) do rio Macacu que determina a proteção de terrenos na faixa complementar de $150 \mathrm{~m}$ de suas margens e seu principal afluente, o rio Guapiaçu (Rio de Janeiro, 2002).

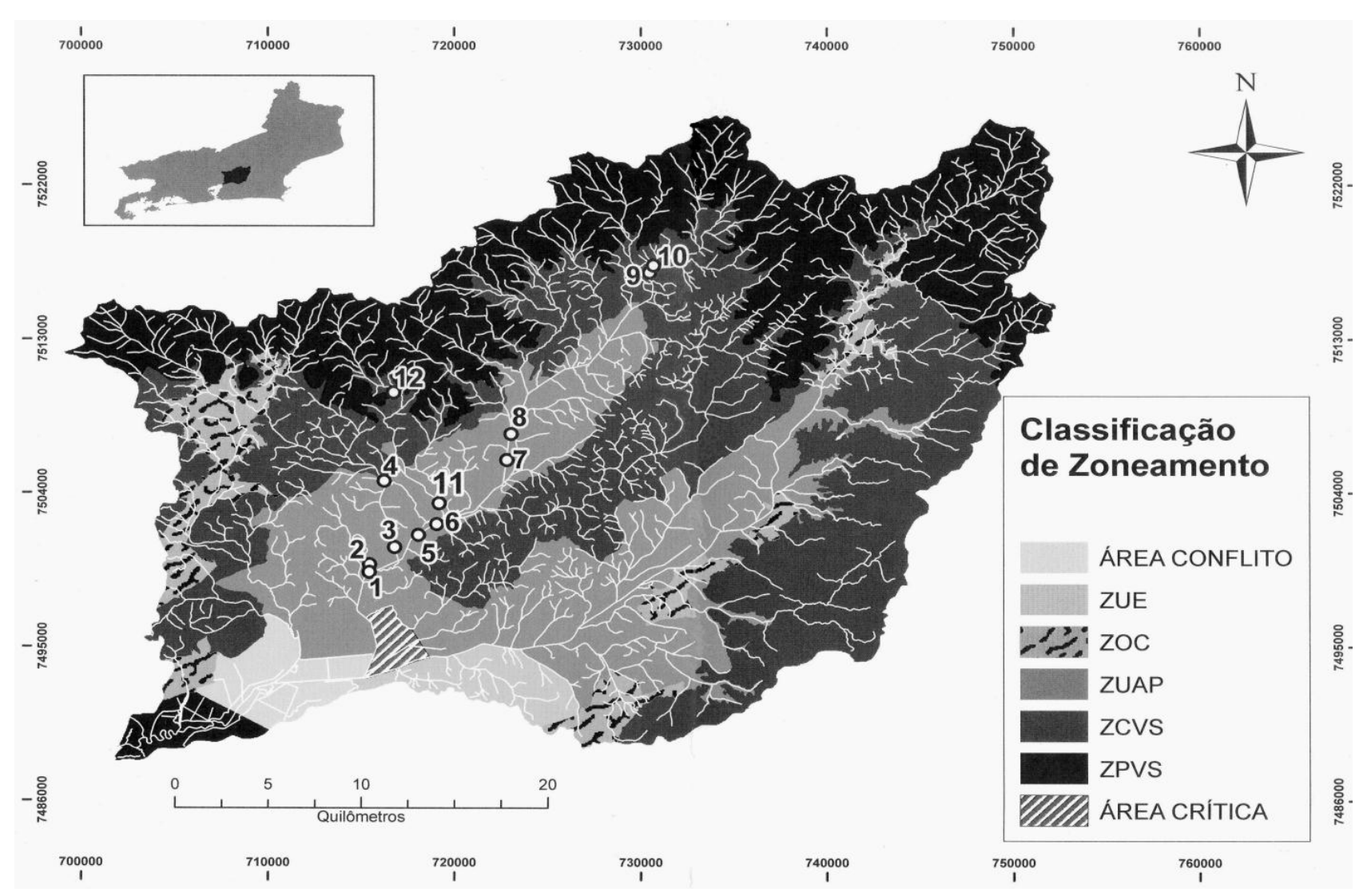

Figura 1. Zoneamento Ambiental da APA proposto pelo Instituto BioAtlântica (2009). Os pontos representam as coletas realizadas durante o projeto, onde ZPVS - Zona de Preservação da Vida Silvestre, ZUAP - Zona de Uso Agropecuário, ZCVS - Zona de Conservação da Vida Silvestre, ZOC Zona de Ocupação Controlada, ZUE - Zona de Uso Especial.

\subsection{Coleta de águas superficiais}

Foram realizadas amostragens bimestrais da água superficial no período de maio de 2008 até junho de 2009 em 12 diferentes pontos, totalizando 72 amostragens em 6 campanhas (Figura 1). A Tabela 1 mostra a localização geográfica dos trechos de rios dentro da área de estudo. 
PEREIRA, P. S.; FERNANDES, L. A. C.; OLIVEIRA, J. L. M.; BAPTISTA, D. F. Avaliação da integridade ecológica de rios em áreas do zoneamento ecológico econômico do complexo hidrográfico Guapiaçu-Macacu, RJ, Brasil. Ambi-Agua, Taubaté, v. 7, n. 1, p. 157-168, 2012. (http://dx.doi.org/10.4136/ambi-agua.762)

Para as análises físicas e químicas foram utilizados frascos de polipropileno e para análise microbiológica, sacos estéreis Whirl-Pak (Nasco). As amostras foram acondicionadas a $4^{\circ} \mathrm{C}$ e transportadas até o laboratório para análise imediata.

Tabela 1. Localização geográfica dos 12 diferentes estações de coleta em 12 estações de coleta, na sub-bacia do rio Guapiaçu.

\begin{tabular}{|c|c|c|c|c|}
\hline Pontos & Nome do rio & ZEE & \multicolumn{2}{|c|}{ Coordenadas geográficas } \\
\hline P1 & Rio Portinho & \multirow{8}{*}{ ZUAP } & W 715478 & S 7499815 \\
\hline $\mathbf{P 2}$ & Rio Portinho & & W 715460 & S 7499369 \\
\hline P3 & Rio Iconha & & W 716820 & S 7500820 \\
\hline $\mathbf{P 4}$ & Rio Iconha & & W 716258 & S 7504708 \\
\hline P5 & Rio Guapiaçu* & & W 718102 & S 7501532 \\
\hline P6 & Rio Guapiaçu* & & W 719085 & S 7502164 \\
\hline P7 & Rio Guapiaçu** & & W 722832 & S 7505919 \\
\hline P8 & Rio Guapiaçu $^{* *}$ & & W 723057 & S 7507448 \\
\hline P9 & Rio Guapiaçu*** & & W 730483 & S 7516884 \\
\hline P10 & Rio Gato ${ }^{* * * *}$ & ZCVS & W 730695 & S 7517321 \\
\hline P11 & Rio Paraíso & ZUAP & W 736359 & S 7503135 \\
\hline P12 & Rio Paraíso & ZCVS & W 716771 & S 7509880 \\
\hline
\end{tabular}

\subsection{Análises físico-químicas e microbiológicas}

As medições de $\mathrm{pH}$, condutividade, sólidos totais dissolvidos (STD) e oxigênio dissolvido (OD) foram executadas no local da coleta utilizando as sondas MPA 210p e MCA 150p (LabConte) e a 550A (YSI). As análises de alcalinidade, dureza e o teor de cloretos foram realizados de acordo com APHA (2005). Para as análises de coliformes totais e termotolerantes foi utilizado o kit Colilert (Idexx).

\subsection{Protocolo de Avaliação Visual (PAV)}

A avaliação visual da área de amostragem foi efetuada por meio do preenchimento do Protocolo de Avaliação Visual (PAV) criado pela agência de proteção ambiental americana (EPA) durante a década de 1980 (Plafkin et al., 1989). O PAV é uma das ferramentas que compõem o Protocolo de Avaliação Rápida que agregam indicadores dos aspectos físicos e biológicos referente à avaliação dos recursos hídricos (Barbour et al., 1999). No PAV são avaliados 10 parâmetros ambientais divididos em caracterização do substrato, condição do canal de água corrente, estabilidade da margem, cobertura e extensão da mata ciliar, substratos disponíveis para fauna bentônica. 
Cada parâmetro recebeu uma pontuação de 0 a 20 para uma condição de ruim a ótima, respectivamente. $\mathrm{O}$ valor final do protocolo de cada trecho foi determinado pela média aritmética dos 10 parâmetros verificados. Assim, foi obtida uma classificação entre as 4 categorias: ótimo, bom, regular ou ruim. As áreas com médias menores que 6,0 foram consideradas como impactadas, ou seja, com uma condição ecológica ruim; as que tiveram médias entre 6,0 e 10,0 foram classificadas como regulares ou intermediárias; as que tiveram médias entre 10,0 e 15,0 estavam boas pelo ponto de vista ambiental; enquanto que as de excelente ou ótima condição tiveram médias superiores a 15,0 pontos.

\subsection{Análise de dados}

Foram realizadas análises estatísticas no Programa Past (Hammer et al., 2001) para a avaliação dos resultados físicos, químicos, microbiológicos e do Protocolo de Avaliação Visual. O teste $U$ de Mann-Whitney $(\mathrm{p}<0,05)$ foi utilizado para avaliar o grau de significância de distinção entre os resultados. A análise multivariada de agrupamento por correlação foi aplicada para comparar os trechos de rios estudados nas duas diferentes Zonas Econômicas Ecológicas.

\section{RESULTADOS E DISCUSSÃO}

A Tabela 2 apresenta os resultados físicos, químicos e microbiológicos entre a Zona de Conservação da Vida Silvestre (ZCVS) e a Zona de Uso Agropecuário (ZUAP). Na área mais restritiva quanto à ocupação e ao uso do solo (ZCVS) foram verificadas concentrações significativamente diferentes desses parâmetros em relação à ZUAP. A ZCVS é caracterizada como uma área que serve como zona de amortização dos impactos à Zona de Preservação da Vida Silvestre (ZPVS) e que juntas devem manter a qualidade de suas águas para o equilíbrio do ecossistema. Em geral, os locais com maior restrição quanto à ocupação e ao uso do solo apresentaram uma melhor qualidade de suas águas.

A Figura 2 indica que houve variação nos valores da condutividade e de sólidos totais dissolvidos (STD) entre a ZUAP e ZCVS. O teste U de Mann-Whitney confirmou a diferença significativa $(\mathrm{p}<0,00001)$ para ambos os parâmetros. A condutividade está relacionada à presença de partículas carregadas eletricamente, logo pode contribuir para a identificação de trechos impactados de uma bacia de drenagem. Este impacto provavelmente é devido ao lançamento de resíduos agrícolas e a entrada de dejetos orgânicos (Pereira et al., 2006). O aumento da concentração de STD normalmente está associado à entrada de partículas inorgânicas na água. Uma das possíveis origens dessas partículas é o uso inadequado da terra como a atividade agropecuária o que leva ao desmatamento e, consequentemente, à erosão das margens dos rios (Custódio e Llamas, 1983).

Os pontos 1 e 2 localizados no rio Portinho (ZUAP) apresentaram os menores valores de oxigênio dissolvido, como mostrado a Figura 3. Esses valores observados estão fora do recomendado para água doce Classe II segundo a resolução Conama 357 (Brasil, 2005). As zonas ZCVS e ZUAP foram significativamente diferentes em relação ao teor de OD e a concentração de coliformes termotolerantes com um valor de "p" igual a 0,001 e 0,004 , respectivamente (Figura 3). O ponto 5 (ZUAP) obteve o maior valor de coliformes termotolerantes provenientes de pastagens em seu entorno e de um assentamento rural local. No entanto neste mesmo trecho foi medida uma concentração OD maior que $6 \mathrm{mg} / \mathrm{L}$ provavelmente devido à declividade do rio. $\mathrm{O}$ uso inadequado do solo para agropecuária reduz a proteção da mata ciliar, o que também acarreta no aumento do processo erosivo das margens, provocando assoreamento e alterações no perfil vertical e na calha do rio. 
PEREIRA, P. S.; FERNANDES, L. A. C.; OLIVEIRA, J. L. M.; BAPTISTA, D. F. Avaliação da integridade ecológica de rios em áreas do zoneamento ecológico econômico do complexo hidrográfico Guapiaçu-Macacu, RJ, Brasil. Ambi-Agua, Taubaté, v. 7, n. 1, p. 157-168, 2012. (http://dx.doi.org/10.4136/ambi-agua.762)

Tabela 2. Estatística descritiva dos parâmetros de qualidade da água analisados em 12 estações amostrais na sub-bacia do rio Guapiaçu.

\begin{tabular}{|c|c|c|c|c|}
\hline Parâmetros & $\begin{array}{c}\text { Áreas da } \\
\mathbf{Z E E}^{\mathbf{1}} \\
\end{array}$ & $\operatorname{Min}^{2}$ & $\operatorname{Max}^{3}$ & Média \\
\hline \multirow{2}{*}{ Oxigênio Dissolvido (mg/L) } & $\mathrm{ZUAP}^{4}$ & 3,3 & 7,6 & 5,9 \\
\hline & $\mathrm{ZCVS}^{5}$ & 5,6 & 8,3 & 6,6 \\
\hline \multirow{2}{*}{$\mathrm{pH}$} & ZUAP & 5,9 & 7,4 & 6,7 \\
\hline & ZCVS & 6,5 & 7,7 & 7,1 \\
\hline \multirow{2}{*}{ Condutividade $(\mu \mathrm{S} / \mathrm{cm})$} & ZUAP & 20,3 & 56,4 & 33,7 \\
\hline & ZCVS & 13,1 & 37,1 & 24,3 \\
\hline \multirow{2}{*}{ Sólidos Totais Dissolvidos (mg/L) } & ZUAP & 9,2 & 26,5 & 16,1 \\
\hline & ZCVS & 5,8 & 17,2 & 11,6 \\
\hline \multirow{2}{*}{ Alcalinidade Total (mg/l CaCO3) } & ZUAP & 9,3 & 20,6 & 16,2 \\
\hline & ZCVS & 9,3 & 16,8 & 14,1 \\
\hline \multirow{2}{*}{ Teor de Cálcio (mg/L) } & ZUAP & 1,6 & 5,5 & 3,1 \\
\hline & ZCVS & 1,6 & 3,3 & 2,6 \\
\hline \multirow{2}{*}{ Teor de Magnésio (mg/L) } & ZUAP & 0,5 & 3,7 & 1,5 \\
\hline & ZCVS & 0,5 & 1,9 & 0,8 \\
\hline \multirow{2}{*}{ Dureza Total (mg/L) } & ZUAP & 6,8 & 21,5 & 13,8 \\
\hline & ZCVS & 6,0 & 14,0 & 9,5 \\
\hline \multirow{2}{*}{ Dureza de $\mathrm{Mg}(\mathrm{mg} / \mathrm{L})$} & ZUAP & 2,0 & 15,6 & 6,1 \\
\hline & ZCVS & 1,9 & 8,0 & 3,3 \\
\hline \multirow{2}{*}{ Dureza Ca (mg/L) } & ZUAP & 2,0 & 13,7 & 7,5 \\
\hline & ZCVS & 1,9 & 8,0 & 3,3 \\
\hline \multirow{2}{*}{ Teor de Cloretos (mg/L) } & ZUAP & 4,3 & 16,6 & 8,9 \\
\hline & ZCVS & 3,9 & 16,6 & 8,2 \\
\hline \multirow{2}{*}{ Coliformes Termotolerantes (NMP/100 mL) } & ZUAP & 0 & 241 & 62,6 \\
\hline & ZCVS & 0 & 58 & 17,5 \\
\hline
\end{tabular}

Nota: ${ }^{1}$ ZEE-zoneamento ecológico-econômico; ${ }^{2}$ Min-mínimo; ${ }^{3}$ Max-máximo; ${ }^{4}$ ZUAP-zona de uso agropecuário; ${ }^{5}$ ZCVS-zona de conservação da vida selvagem.

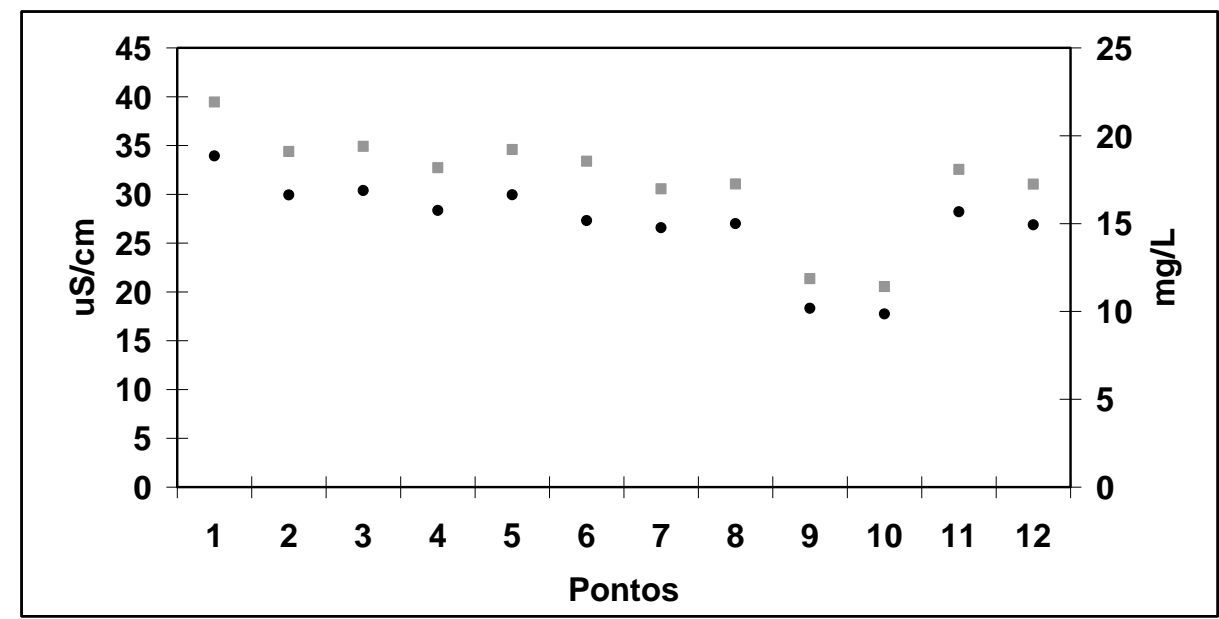

Figura 2. Resultados da condutividade $(\mu \mathrm{S} / \mathrm{cm})$ ( $\square$ ) e sólidos totais dissolvidos $(\mathrm{mg} / \mathrm{L})(\bullet)$ nos doze diferentes trechos de rios amostrados, no período de maio de 2008 até junho de 2009. 


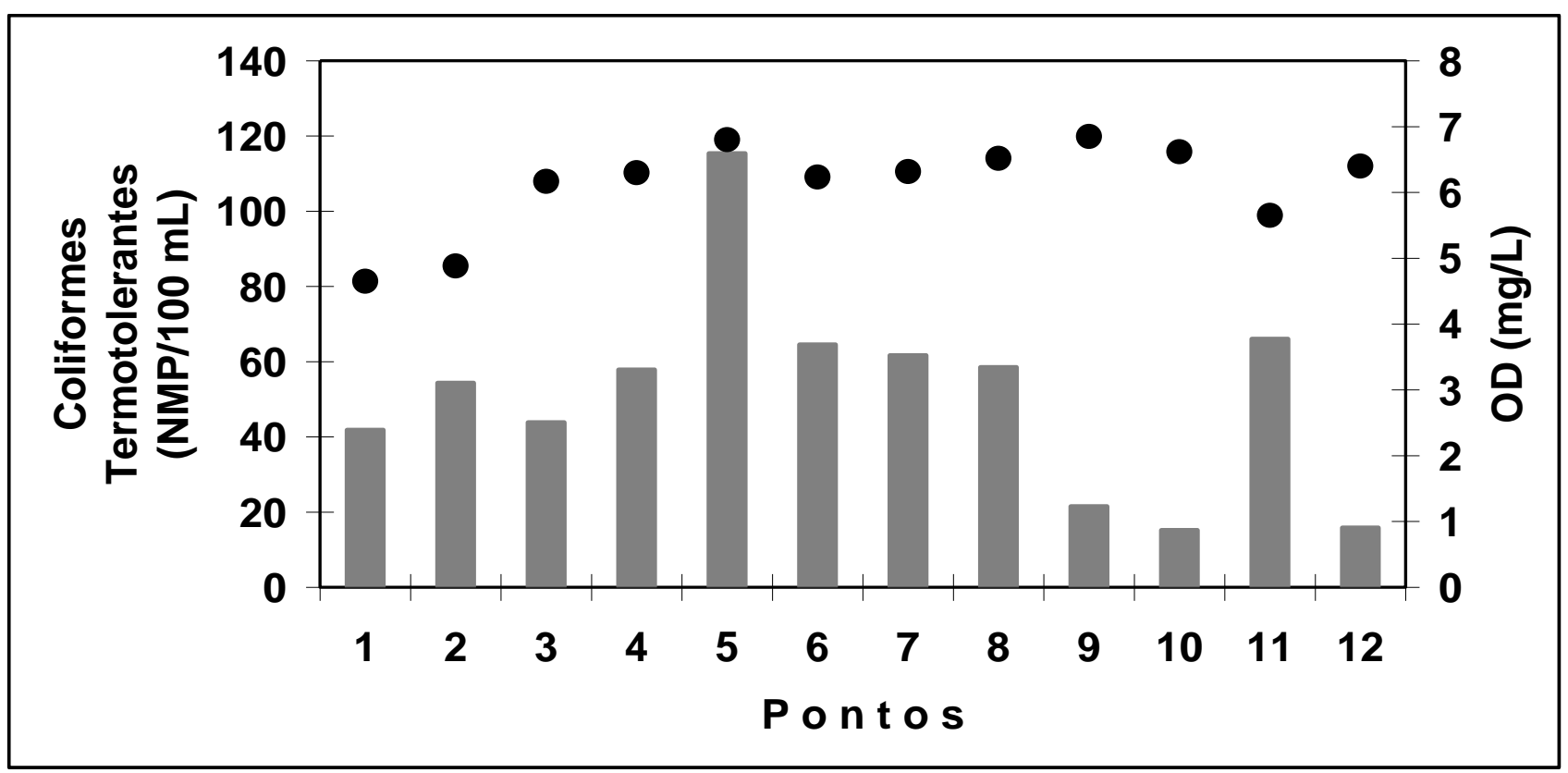

Figura 3. Resultados de oxigênio dissolvido $(\bullet)$ e de coliformes termotolerantes (barra) em doze diferentes trechos de rios amostrados, no período de maio de 2008 até junho de 2009.

A classificação da paisagem que foi realizada usando-se o Protocolo de Avaliação Visual (PAV) enquadrou a ZUAP em duas diferentes categorias paisagísticas. Os pontos de 1 a 6 obtiveram uma classificação ruim, enquanto que os pontos 7,8 e 11 apresentaram uma condição ecológica regular conforme a Tabela 3. Esta condição de subdivisão da característica da qualidade ambiental do rio não pode ser detectada por meio das análises físicas, químicas e microbiológicas embora estes trechos estejam dentro de uma mesma zona (ZUAP). Este resultado sugere que os trechos de rios que ainda se encontram em um estágio devam receber uma atenção especial pelos gestores da APA, pois são zonas de transição ecológica tendendo a atingir um estágio ruim. Os trechos de rios localizados na ZCVS (9, 10 e 12) apresentaram uma boa integridade ecológica pelo PAV. Estes resultados corroboraram com o modelo proposto de ZEE pelo Instituto Bioatlântica. Portanto, o PAV pode ser uma ferramenta útil no diagnóstico da integridade ecológica da paisagem como foi sugerido por outros autores (Callisto et al., 2002; Rodrigues et al., 2008, 2010).

Segundo o PAV, na ZCVS foram encontrados substratos favoráveis para colonização da biota aquática, indicando a presença de microhabitats que fornecem suporte alimentar e abrigo para manutenção da vida aquática, conforme previsto para está área. A alteração desses substratos pode prejudicar a formação de microhabitats e, com isso, organismos como macroinvertebrados bentônicos podem ser afetados, pois dependem desses microambientes. Este elemento fisiográfico é um fator importante para a manutenção da diversidade biológica do rio e da qualidade de suas águas.

$\mathrm{Na}$ ZUAP, os pontos 3, 4 e 6 encontravam-se em áreas retificadas dos rios. Esta característica difere da condição natural de um rio que forma meandros característicos desta área de planície costeira. A retificação de um rio acarreta no aumento da velocidade de sua correnteza provocando a homogeneização de seu leito devido ao assoreamento de material particulado. 
PEREIRA, P. S.; FERNANDES, L. A. C.; OLIVEIRA, J. L. M.; BAPTISTA, D. F. Avaliação da integridade ecológica de rios em áreas do zoneamento ecológico econômico do complexo hidrográfico Guapiaçu-Macacu, RJ, Brasil. Ambi-Agua, Taubaté, v. 7, n. 1, p. 157-168, 2012. (http://dx.doi.org/10.4136/ambi-agua.762)

Com isso estes trechos podem ser facilmente inundados, o que facilita seu uso no plantio, mas piora a sua qualidade ambiental e, consequentemente, reduz a qualidade de suas águas e altera a sua diversidade biológica (Lucas e Cunha, 2007).

Os valores atribuídos para mata ciliar na ZCVS foram acima de 10 pontos (exceto o ponto 12) mostrando uma boa integridade ecológica, diferente dos trechos da ZUAP. A ZUAP quase não apresentou áreas com extensão de mata ciliar, com exceção do ponto 7 que mantinha cerca de 30 metros de largura e uma vegetação arbórea em estágio de recuperação. A redução de mata ciliar reduz a retenção de sedimentos que são arrastados ao rio pelo escoamento superficial do seu entorno (Curvello et al., 2008; Santos e Cardoso, 2007; Coelho et al., 2011). Com isso, esses trechos ficam assoreados, reduzindo sua profundidade e, portanto, a qualidade de suas águas.

Pelos resultados físicos, químicos, microbiológicos e os aspectos visuais da integridade da paisagem (PAV) foi feita uma análise de agrupamento mostrada na Figura 4. Este dendrograma indica que existe uma similaridade de 60\% entre as áreas estudadas (ZUAP e ZCVS) desta sub-bacia pelo índice de correlação. Oliveira (2009) que fez um estudo nesta mesma área incluindo a Zona de Preservação da Vida Silvestre (ZPVS) e a Zona de Ocupação Controlada (ZOC) e encontrou padrões semelhantes aos nossos. Portanto, os resultados obtidos estão coerentes com o modelo de zoneamento proposto para esta região.

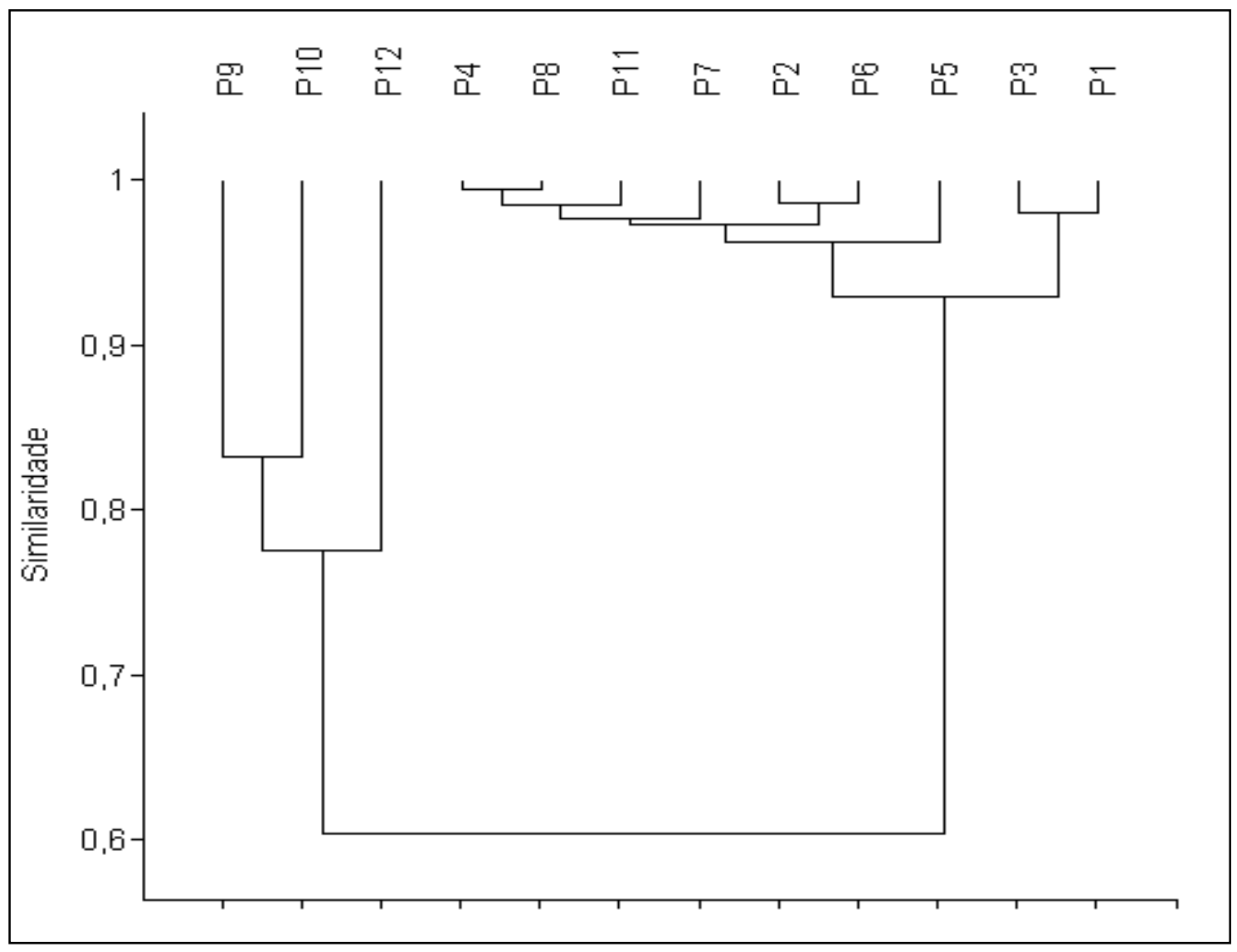

Figura 4. Dendrograma mostrando o agrupamento dos doze diferentes trechos de rios amostrados. 
PEREIRA, P. S.; FERNANDES, L. A. C.; OLIVEIRA, J. L. M.; BAPTISTA, D. F. Avaliação da integridade ecológica de rios em áreas do zoneamento ecológico econômico do complexo hidrográfico Guapiaçu-Macacu, RJ, Brasil. Ambi-Agua, Taubaté, v. 7, n. 1, p. 157-168, 2012. (http://dx.doi.org/10.4136/ambiagua.762)

Tabela 3. Resultado do Protocolo de Avaliação Visual (PAV) dos diferentes trechos de rios.

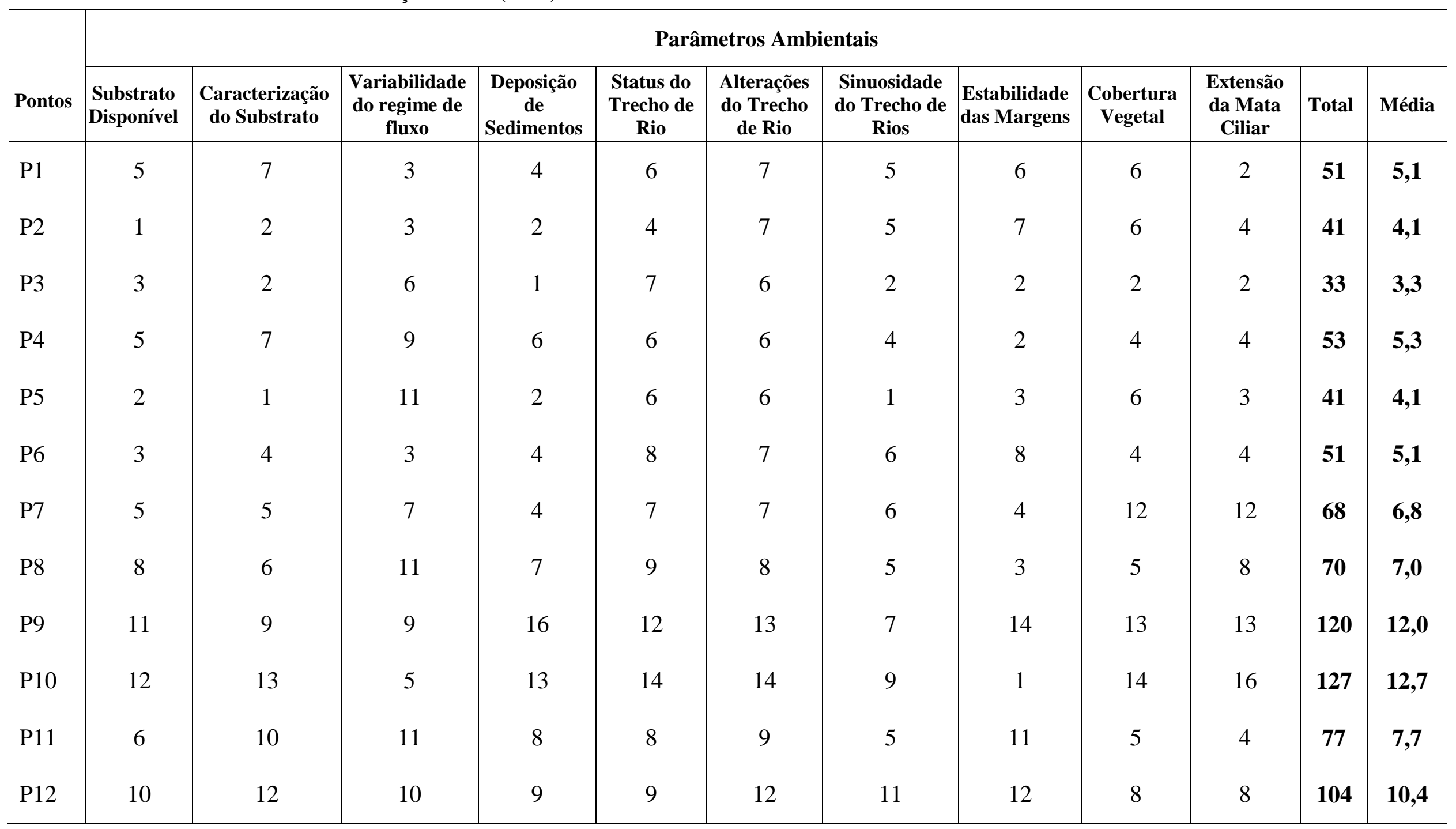

Nota: Escalas de Classificação: 0 a 6,0 - ruim (impactado); 6,0 a 10,0 - regular; 10,0 a 15,0 - boa; 15,0 a 20,0 - ótima. 


\section{CONCLUSÕES}

Este estudo permitiu as seguintes conclusões:

As áreas mais restritivas quanto à ocupação e ao uso do solo (ZCVS) tiveram uma melhor qualidade da água do que os trechos das áreas menos restritivas (ZUAP) em função dos parâmetros físicos, químicos e microbiológicos avaliados.

O PAV corroborou como os parâmetros físicos, químicos e microbiológicos na avaliação dos trechos da ZCVS, no entanto somente a avaliação fisiográfica foi capaz de distinguir as áreas de transição das impactadas da ZUAP.

O PAV foi mais sensível na avaliação da integridade ecológica de uma área do que os métodos de avaliação físicos, químicos e microbiológicos.

\section{AGRADECIMENTOS}

Os autores gostariam de agradecer a ONG Instituto BioAtlântica, pelo financiamento do projeto e confecção do mapa, o apoio financeiro do CNPq (PROEP/FIOCRUZ) e a FAPERJ pela bolsa de estudo. Os colegas do LAPSA/IOC/FIOCRUZ pela realização deste estudo, em especial aos técnicos Valdinei Valin e Denise Borges do Laboratório de Avaliação e Promoção da Saúde Ambiental pela realização das análises.

\section{REFERÊNCIAS}

AB'SABER, A. Zoneamento ecológico e econômico da Amazônia: questões de escala e método. Revista Estudos Avançados, v.3, n. 5, p. 4-20, 1989.

ACSELRAD, H. Zoneamento ecológico-econômico e a multiplicidade de ordens socioambientais na Amazônia. Novos Cadernos Naea, v. 3, n. 2, p. 5-15, 2000.

AMERICAN PUBLIC HEALTH ASSOCIATION - APHA. Standard methods for the examination of water and wastewater. $20^{\text {th }}$ ed. Washington, DC, 2005.

BARBOUR, M. T.; GERRITSEN, J.; SNYDER, B. D.; STRIBLING, J. B. Rapid bioassessment protocols for use in streams and wadeable rivers: periphyton, benthic macroinvertebrates and fish. 2. ed. Washington: EPA, 1999. $339 \mathrm{p}$.

BRASIL. Ministério do Meio Ambiente. Resolução Conama no 357 de 17 de março de 2005. Dispõe sobre a classificação dos corpos de água e diretrizes ambientais para o enquadramento, bem como estabelece as condições e padrões de lançamento de efluentes, e dá outras providências. Disponível em: <http://www.mma.gov.br/ port/conama/res/res05/res35705.pdf>. Acesso em: 19 mar. 2012.

CABRAL, D. C.; FISZON, J. T. Padrões sócio-espaciais de desflorestamento e suas implicações para a fragmentação florestal: estudo de caso na Bacia do Rio Macacu, RJ. Scientia Florestalis, n. 66, p. 13-24, 2004.

CALLISTO, M. et al. Aplicação de um protocolo de avaliação rápida da diversidade de habitats em atividades de ensino e pesquisa (MG-RJ). Acta Limnologica Brasiliensis, v. 14, n. 1, p. 91-98, 2002. 
CAMPANHA, M. B.; MELO, C. A., MOREIRA, A. B.; FERRARESE, R. F. M. S.; TADINI, A.M.; GARBIN, E. V. et al. Variabilidade espacial e temporal de parâmetros físicoquímicos nos rios Turvo, Preto e Grande no estado de São Paulo, Brasil. Revista Química Nova, v. 33, n. 9, p. 1831-1836, 2010.

CARVAlHO, A. R.; SCHLITTLE, F. H. M.; TORNISIELO, V. L. Relações da atividade agropecuária com parâmetros físicos químicos da água. Revista Química Nova, v. 23, p. $618-622,2000$.

COELHO, R. C. T. P.; BUFFON, I.; GUERRA, T.; Influência do uso e ocupação do solo na qualidade da água: um método para avaliar a importância da zona ripária. Revista Ambiente \& Água, v. 6, n. 1, p. 104-117, 2011. http://dx.doi.org/10.4136/ambiagua. 178

CORADI, P. C.; FIA R.; PEREIRA-RAMIREZ, O. Avaliação da qualidade da água superficial dos cursos de água do município de Pelotas-RS, Brasil. Revista Ambiente \& Água, v. 4, n. 2, p. 46-56, 2009. http://dx.doi.org/10.4136/ambi-agua.86

CURVELlO, R. T.; BATISTA, G. T.; TARGA, M. S. Estudo dos impactos da ocupação humana na microbacia do rio Batedor na Serra da Mantiqueira no município de Cruzeiro, SP, Brasil. Revista Ambiente \& Água, v. 3, n. 1, p. 91-107, 2008. http://dx.doi.org/10.4136/ambi-agua.45

CUSTÓDIO, E.; LLAMAS, M. R. Hidrologia Subterrânea. Tomo I e II. Barcelona: Ómega, 1983. $2350 \mathrm{p}$.

HAMMER, O.; HARPER, D. A. T.; RYAN, P. D. PAST: paleontological statistics software package for education and data analysis. Palaeontologia Electronica, v. 4, n. 1, p. 9, 2001.

HELDER, C. Subsídios para gestão dos recursos hídricos das bacias hidrográficas dos rios Macacu, São João, Macaé e Macabu. Rio de Janeiro: Secretaria do Meio Ambiente, 1999.

INSTITUTO BIOATLÂNTICA. Plano de manejo: APA da bacia do rio Macacu: proposta. In: Projeto entre Serras e Águas. Rio de Janeiro: 2009. Disponível em: <http://pib.socioambiental.org/anexos/6658_20091117_095531.pdf>. Acesso: 19 mar. 2012.

JAPAN INTERNACIONAL COOPERATION AGENCY - JICA. The study of recuperation of the Guanabara Bay ecosystem. Supporting Report. Tokyo: Kokusai Kogyo, 1994. Vol. 1-4.

LUCAS, L. M.; CUNHA, S. B. Rede de drenagem urbana em área tropical: mudanças na morfologia do canal e níveis de poluição das águas - Rio dos Macacos, Rio de Janeiro. GEOUSP - Espaço e Tempo, n. 22, p. 39-64, 2007.

MACHADO, C. J. S.; KLEIN, H. E. Água, doença, saúde e arcabouço institucional-legal: por uma gestão integrada das águas do Estado do Rio de Janeiro. Revista Rio de Janeiro, n. 11, p. 13-38, 2003. 
OLIVEIRA, R. B. Desenvolvimento de um índice multimétrico rápido baseado na comunidade de macroinvertebrados bentônicos para avaliação da integridade ecológica de riachos do complexo Guapiaçu-Macacu, RJ. 2009. 107f. Dissertação (Mestrado em Ecologia) - PPGE, Universidade Federal do Rio de Janeiro, Rio de janeiro, 2009.

PEDREIRA, B. C. C. G.; FIDALGO, E. C. C.; ABREU, M. B. Mapeamento do uso e cobertura da terra da bacia hidrográfica do rio Guapi-Macacu, RJ. In: SIMPÓSIO BRASILEIRO DE SENSORIAMENTO REMOTO, 14., 25-30 abril 2009, Natal. Anais... São José dos Campos: INPE, 2009. p. 2111-2118.

PEREIRA, M. R, ALVES, C., MARTINS, F., MACHADO, M., LOPES, S. A Qualidade da Água na Bacia Hidrográfica do Rio Sôrdo (Norte de Portugal). Geonovas, n. 20, p. 8793, 2006.

PLAFKIN, J. L.; BARBOUR, M. T.; PORTER, K. D.; GROSS, S. K.; HUGHES, R. M. Rapid bioassessment protocols for use in streams and rivers: benthic macroinvertebrates and fish. Washington: U.S. Environmental Protection Agency, EPA444/4-89-001, 1989.

RIO DE JANEIRO (Estado). Lei Estadual no 4018 de 05 de dezembro de 2002. Cria a área de proteção ambiental da bacia do rio macacu e determina providências para a defesa da qualidade da água. Disponível em: <http://www.inea.rj.gov.br/legislacao/docs/ 4018.pdf>. Acesso em: 19 mar. 2012.

RODRIGUES, A. S. L.; MALAFAIA, G.; CASTRO, P. T. A. Protocolos de avaliação rápida de rios e a inserção da sociedade no monitoramento dos recursos hídricos. Revista Ambiente \& Água, v. 3, n. 3, p. 143-155, 2008. http://dx.doi.org/10.4136/ambi-agua.68

RODRIGUES, A. S. L.; MALAFAIA, G.; CASTRO, P. T. A. A importância da avaliação do habitat no monitoramento da qualidade dos recursos hídricos: uma revisão. Revista Saúde e Biologia, v. 5, n.1, p. 26-42, 2010.

SANTOS, A. F.; CARDOSO, L. G. Delimitação das áreas de preservação Permanente (Mata ciliar) da microbacia hidrográfica do Ribeirão Faxinal, Botucatu-SP. In: SEMINÁRIO DE RECURSOS HÍDRICOS DA BACIA HIDROGRÁFICA DO PARAÍBA DO SUL, 1., 2007, Taubaté. O eucaplipto e o ciclo hidrológico. Anais... Taubaté: IPABHi, 2007. p. 231-235.

SARCINELLI, O.; MARQUES, J. F.; ROMEIRO A. R. Custo de adequação ambiental das áreas de vegetação ripária: estudo de caso na microbacia do Córrego Oriçanguinha, SP. Revista Informações Econômicas, v. 38, n. 10, p. 70-79, 2008.

TUCCI, C. E. M. Hidrologia: ciência e aplicação. Porto Alegre: UFRGS, 2002. 248 p.

TUNDISI, J. G. Água no século XXI: enfrentando a escassez. São Carlos: RIMA, 2003. $247 \mathrm{p}$.

VASCO, A. N.; BRITTO, F. B.; PEREIRA, A. P. S.; MÉLlO JÚNIOR, A. V. M.; GARCIA, C. A. B.; NOGUEIRA, L. C. Avaliação espacial e temporal da qualidade da água na sub-bacia do rio Poxim, Sergipe, Brasil. Revista Ambiente \& Água, v. 6, n. 1, p. 118130, 2011. http://dx.doi.org/10.4136/ambi-agua.178 